\title{
Risk Assessment of a Cryogenic Globe Valve by Using Combined Group Decision and Fuzzy Comprehensive Evaluation
}

\author{
Yi Lu, ${ }^{1,2}$ Jianming Zheng $\mathbb{D}^{1}{ }^{1}$ Chen Zhao, ${ }^{1}$ and Gang Ren ${ }^{2}$ \\ ${ }^{1}$ Xi'an University of Technology, School of Mechanical and Precision Instrument Engineering, Xi'an 710048, China \\ ${ }^{2}$ The Boiler \& Pressure Vessel Safety Inspection Institute of He nan Province, Zhengzhou 450016, China \\ Correspondence should be addressed to Jianming Zheng; zjm@xaut.edu.cn
}

Received 30 November 2020; Revised 11 February 2021; Accepted 15 February 2021; Published 24 February 2021

Academic Editor: Xindong Peng

Copyright (c) 2021 Yi Lu et al. This is an open access article distributed under the Creative Commons Attribution License, which permits unrestricted use, distribution, and reproduction in any medium, provided the original work is properly cited.

When using the risk priority number method to perform a criticality analysis of the failure modes of mechanical products, different subjective factors exist on the part of experts regarding the severity level (S), occurrence probability level (O), and failure modes. The estimation of the detection difficulty level (D) is different, which causes the problem of inaccurate evaluation of the criticality of failure modes. This study proposes a harm analysis method that combines group decision-making and fuzzy comprehensive evaluation. The similarity and difference between an individual expert and the group are used in group decisionmaking to assign the weight of the expert, thus reducing the influence of subjective factors on the evaluation results. On the basis of the fuzzy comprehensive evaluation method, the weight of three risk elements $(\mathrm{S}, \mathrm{O}$, and $\mathrm{D})$ is determined, and a hazard ranking of the interval obtained by group decision-making is performed. The CA (criticality analysis) improvement method is used to analyze the hazard posed by low-temperature shut-off valves. Results indicate that this method can effectively assess the weak links of the low-temperature shut-off valves and improve the accuracy of the hazard evaluation in comparison with the risk priority coefficient method.

\section{Introduction}

Cryogenic shut-off valve is one of the most commonly used valves in cryogenic systems. Cryogenic shut-off valves are utilized at different temperatures in many areas, such as air separation and LNG transportation. Their reliability determines the safety of the entire cryogenic system. Therefore, increasingly strict requirements for the reliability of cryogenic stop valves are being put forward. A hazardous analysis of the various failure modes of cryogenic shut-off valves must be conducted to improve the reliability of these valves and identify their weak links. The risk priority number (RPN) method can intuitively reveal the degree of harm of the product failure mode, and its results are widely used in the formulation and implementation of improvement measures. Qiang [1] utilized RPN analysis to evaluate the failure modes of precision gears; they judged and ranked the modes by optimizing each process parameter of the gear manufacturing process. However, the weight distribution of the three risk elements was not provided by experts. Dai et al. [2] assigned weights to the hazard degree, probability of occurrence, and detection difficulty of an electro-hydraulic servo valve and used RPN analysis to determine the relative hazard levels of the failure modes and sort them to improve reliability further. The level of protection has a positive value, but it does not consider the impact of the subjective factors of experts on the valuation. Chang [3] proposed a general RPN method that combines the ordered weighted geometric average operator and decision test to conduct risk assessment of thin-film transistor liquid-crystal displays and obtain the product failure risk data. Chen et al. [4] studied the manufacturing process of crystal oscillators, with focus on risk analysis of critical quality processes, and determined the best priority to improve process quality. Dey et al. [5] proposed a systematic method for establishing the standard equation of RPN measurement on the basis of the logistic regression method using interval numbers. Through a case study, they found that effective risk analysis by the proposed 
method fails in large-capacity electric submersible pumps in power plants. Renjith et al. [6] introduced a method for correcting fuzzy RPN to identify the consequences of component failures in complex systems, such as liquefied natural gas storage, and determine the importance of each failure mode to system performance. Catelani et al. [7] performed risk assessment during the turbine design stage by using the priority ranking of RPN. Their aim was to reduce machine downtime and operation and maintenance costs and ensure service continuity.

In summary, the following problems are encountered in RPN calculation. First, the subjective factors of experts exert a great influence on the weight of experts. Second, the results of the weight distribution of the three risk elements directly affect the accuracy of judging the harmfulness of the failure mode.

This study uses an analysis method that combines group decision-making and fuzzy comprehensive evaluation and fully considers the influence of the subjective factors of experts. Multiple experts give an evaluation interval. Given that the similarity and difference between individual experts and groups are used, the distribution of expert weights is reasonable. The obtained group interval is fuzzy-evaluated via fuzzy comprehensive evaluation, and the abovementioned method is used to analyze the hazard posed by each failure mode of a cryogenic shut-off valve. As a result, the weakness of the cryogenic shut-off valve is effectively assessed, and the evaluation of hazard accuracy is improved.

\section{RPN Calculation Method}

RPN often selects the product of the severity level (S) of the failure mode, the probability level $(\mathrm{O})$, and the detected difficulty level (D) as the judgment basis. The greater the RPN value of the failure mode is, the more harmful the fault is. The calculation formula is as follows:

$$
\mathrm{RPN}=S \times O \times D .
$$

According to the national standard G/JB Z1391-2006, the severity level $(\mathrm{S})$, occurrence probability level $(\mathrm{O})$, and detected difficulty level (D) of a failure mode can be divided into 10 scores or 5 score levels. The severity level is used to evaluate the final degree of influence of a certain failure mode, the occurrence probability level is the possibility of a failure mode actually occurring, and the detected difficulty level represents the difficulty of detection during use. The higher the values of the evaluation factors are, the greater the harmful effect of the failure mode is on the product. The specific scoring standards are shown in Tables 1 and 2 .

It can be seen from the above discussion that the evaluation of the risk priority coefficient method is simple and intuitive, the RPN value in each failure mode can be calculated separately according to the formula combined with the expert score; however, it still has limitations. (1) In the risk priority coefficient method, the evaluation values for the severity level (S), occurrence probability level $(\mathrm{O})$, and detected difficulty level (D) should be a set of evaluations given by an expert or several experts' value. However, it is difficult to unify expert opinions due to the different levels of experience and professional expertise of each expert. In fact, the evaluation value is multiple sets of evaluation values given by multiple experts. (2) In the RPN calculation, the evaluation value given by the expert is represented by an integer from 1 to 10 . However, in fact, the estimated value is difficult to be represented by an exact number among these 10. (3) In the RPN calculation, different combinations of $S$, $\mathrm{O}$, and $\mathrm{D}$ values may get the same RPN value or different failure modes have the same $\mathrm{S}, \mathrm{O}$, and $\mathrm{D}$ values, so the obtained RPN value is also the same, but the failure risk corresponding to RPN is different. Therefore, to overcome the abovementioned shortcomings, this paper proposes a method of combining group decision-making and fuzzy comprehensive evaluation.

\section{RPN Analysis Based on the Combination of Group Decision and Fuzzy Comprehensive Evaluation}

3.1. Principle of RPN Analysis Combining Group Decision and Fuzzy Comprehensive Evaluation. Group decision-making aggregates the preferences of each member of the group for certain things into group preferences so that the group can rank all things in their order of pros and cons [8]. This procedure can effectively avoid the impact of individual differences on the results. The expert scoring system used in this study is a typical group decision-making process $[9,10]$. Therefore, using the similarity and difference between individual experts and groups in group decision-making to assign expert weights can effectively solve the problem of excessive individual subjective factors. The results of the group evaluation interval can also be obtained. Fuzzy comprehensive evaluation is a comprehensive evaluation method based on fuzzy mathematics. According to membership theory in fuzzy mathematics, this method transforms qualitative evaluation into quantitative evaluation. Using fuzzy comprehensive evaluation to evaluate the abovementioned group interval can produce clear results and strong systemicity, which are helpful in solving problems that are difficult to quantify. In summary, this study uses a method that combines group decision-making and fuzzy comprehensive evaluation to analyze the failure mode. The specific principles are discussed below.

In accordance with the failure mode and impact (FMEA), the causes, impact, and severity of failure, detection, and other factors are analyzed. The expert scoring side is determined, and the failure scoring interval is established according to the similarity between an individual expert and the group in group decision-making. The difference degree is used to determine the expert weight value. On the basis of the comprehensive fuzzy evaluation, the weight distribution of the three risk elements in RPN and the membership degree of the expert comprehensive evaluation interval are determined. Then, the RPN value is obtained. The specific steps are shown in Figure 1. 
TABLE 1: Severity rating scale.

\begin{tabular}{ccc}
\hline Grade & Severity rating & Severity definition \\
\hline 5 & 9,10 (disaster) & Causes death or product destruction and major environmental damage \\
4 & 7,8 (deadly) & Causes serious injury to personnel or major losses that lead to mission failure and serious product damage \\
3 & 5,6 & Causes moderate injury or economic loss to personnel, mission delay, or moderate damage to products \\
& $($ moderate) & Not enough to cause personal injury, mild economic loss, or mild product damage but can cause unplanned \\
& 3,4 (mild) & maintenance or repair \\
1 & 1,2 (no effect) & No impact on product function \\
\hline
\end{tabular}

TABLE 2: Occurrence rate and detection difficulty rating scale.

\begin{tabular}{ccccc}
\hline \multicolumn{2}{c}{ Number of ratings } & \multicolumn{2}{c}{ Probability O } & \multicolumn{2}{c}{$\begin{array}{c}\text { Detection difficulty D } \\
\text { Grade }\end{array}$} & Number of ratings & Probability of failure mode & Reference range of incidence $\left(\mathrm{P}_{\mathrm{m}}\right)$ & Definition of detection difficulty \\
\hline 1 & 1,2 & Extremely low & $\mathrm{P}_{\mathrm{m}} \leq 10^{-6}$ & Extremely low \\
2 & 3,4 & Lower & $10^{-6}<\mathrm{P}_{\mathrm{m}} \leq 10^{-4}$ & Lower \\
3 & 5,6 & Medium & $10^{-4}<\mathrm{P}_{\mathrm{m}} \leq 10^{-2}$ & Medium \\
4 & 7,8 & High & $10^{-2}<\mathrm{P}_{\mathrm{m}} \leq 10^{-1}$ & High \\
5 & 9,10 & Very high & $10^{-1} \leq \mathrm{P}_{\mathrm{m}}$ & Very high \\
\hline
\end{tabular}

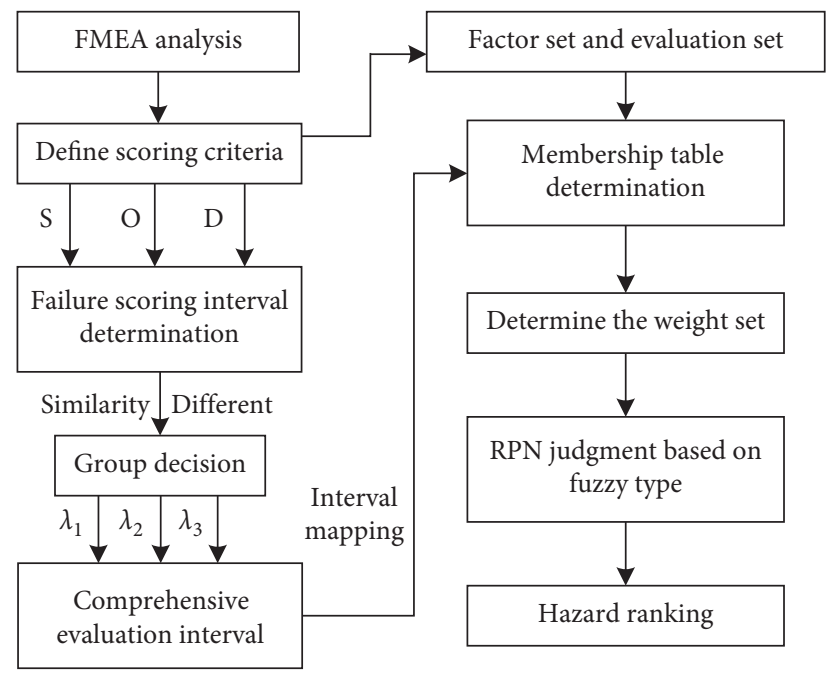

FIGURE 1: RPN analysis process combining group decision-making and fuzzy comprehensive evaluation.

\subsection{Determination of Expert Weight and Failure Mode} Comprehensive Evaluation Interval. In RPN analysis, the method of group decision-making is used to determine the weight of experts. A set of interval numbers are given by multiple experts [6], and the individual evaluation results of the experts are compared with the group evaluation results. The closer the expert is, the higher the credibility is [11]. Therefore, the value assigned to the weight should also be large. The specific method is as follows.

Definition 1. Let $R$ be a real-number field and closed interval $\left[x^{l}, x^{u}\right]$ be the interval number. Note that $x=\left[x^{l}, x^{u}\right]$, where $x^{l}$ and $x^{u}$ are the left and right endpoints of the interval number, respectively, and $\mathrm{x}^{\mathrm{l}}, \mathrm{x}^{\mathrm{u}} \in \mathrm{R}$.
Definition 2. Consider two interval number vectors $\mathrm{X}=\left(\mathrm{x}_{1}, \mathrm{x}_{2}, \cdots \mathrm{x}_{\mathrm{n}}\right)$ and $\mathrm{Y}=\left(\mathrm{y}_{1}, \mathrm{y}_{2}, \cdots \mathrm{y}_{\mathrm{n}}\right)$, where $\mathrm{x}_{\mathrm{i}}=\left[\mathrm{x}_{i}^{L}\right.$, $\left.\mathrm{x}_{i}^{U}\right]$ and $\mathrm{y}_{\mathrm{i}}=\left[\mathrm{y}_{i}^{L}, \mathrm{y}_{i}^{U}\right]$. Their inner product is defined as

$$
<X, Y>=\sum_{i=1}^{n}\left(x_{i}^{L} y_{i}^{L}+x_{i}^{U} y_{i}^{U}\right) .
$$

Definition 3. Log interval $a=\left[a^{l}, a^{u}\right]$ and $b=\left[b^{l}, b^{u}\right]$ and call $D(a, b)=\left|a^{l}-b^{l}\right|+\left|a^{u}-b^{u}\right|$ for their distance.

For failure mode $i$, the cosine of the angle between the evaluation vectors of experts $A$ and expert $B$ is

$$
\eta_{i}=\cos (\theta)=\frac{\left\langle r_{i}(A), r_{i}(B)\right\rangle}{\sqrt{\left\langle r_{i}(A), r_{i}(A)>\left\langle r_{i}(B), r_{i}(B)\right\rangle\right.}},
$$


where $r_{i}(A)=\left(r_{i S}(A), r_{i O}(A), r_{i D}(A)\right)$ indicates expert $k$ 's evaluation value of $\mathrm{S}, \mathrm{O}$, and $\mathrm{D}$ in the failure mode.

The similarity between expert $A$ and other experts regarding the evaluation value of failure mode $i$ is

$$
u_{i}(A)=\frac{\eta_{i}(A)}{\sum_{A=1}^{d} \eta_{i}(A)}
$$

where $\eta_{i}(A)=\sum_{=1}^{d} \eta_{i}(A, B)-1$ and $d$ is the number of experts participating in the evaluation.

The mean vector of the expert group under failure mode $i$ is

$$
\overline{r_{i j}}=\frac{1}{d} \sum_{A=1}^{d} r_{i j}(A)=\left[\frac{1}{d} \sum_{A=1}^{d} r_{i j}^{L}(A), \frac{1}{d} \sum_{A=1}^{d} r_{i j}^{U}(A)\right] .
$$

According to Definition 3, the difference between expert $A$ and other experts in terms of the evaluation value of failure mode $i$ is

$$
\delta_{i}(A)=\frac{\sigma_{i}(A)}{\sum_{A=1}^{d} \sigma_{i}(A)}
$$

where $\sigma_{i}(A)=\sum_{j=1}^{3} \sigma_{i j}(A)$ and $\sigma_{i j}(A)=D\left(r_{i j}(A), \overline{r_{i j}}\right)$.

Considering the similarity and difference of expert evaluation, the weight of experts is defined as

$$
\lambda_{i}(A)=\frac{u_{i}(A)\left(1-\delta_{i}(A)\right)}{\left[1-\sum_{A=1}^{d} u_{i}(A) \delta_{i}(A)\right]} .
$$

Therefore, the weight of each expert can be used to obtain the comprehensive evaluation value of each factor in failure mode $i$ as follows:

$$
Z_{i j}=\sum_{A=1}^{d} \lambda_{i}(A) \cdot r_{i j}(A)=\left[\sum_{A=1}^{d} \lambda_{i}(A) \cdot r_{i j}^{L}(A), \sum_{A=1}^{d} \lambda_{i}(A) \cdot r_{i j}^{U}(A)\right] .
$$

Using group decision-making, the method of combining similarity and difference of expert evaluation is used to determine the weight of each expert in group decisionmaking, which overcomes the influence of factors such as the experts' experience level and individual preference in the risk priority coefficient method. At the same time, because the expert gives the evaluation interval, the problem of inaccurate evaluation caused by the expert's fixed value is also avoided. However, it is difficult to intuitively discover the severity of each failure mode through the comparison of intervals. Therefore, combined with comprehensive fuzzy evaluation and de-fuzzy processing, qualitative analysis is transformed into quantitative comparison.

\subsection{Fuzzy Comprehensive Evaluation and Determination of} $R P N$ Value. Generally, fuzzy comprehensive evaluation consists of four parts. First, the factor and evaluation sets are established to clarify the factors and evaluation standards that affect the system. Second, the degree of membership is determined to obtain the evaluation matrix of different factors. Third, the weights are determined to achieve the relative weights between different factors. Lastly, fuzzy comprehensive evaluation is performed to obtain the severity of each failure mode.

3.3.1. Determination of Factor and Evaluation Sets. In accordance with the actual investigation and analysis of the failure mode, the system factor set is determined as $U=$ \{severity level (S), occurrence probability level (O), and detected difficulty level (D)\}. The evaluation index system has been analyzed in the previous section. It is divided into five levels, that is, $\mathrm{V}=\{1,2,3,4,5\}$.

3.3.2. Determination of Membership. The traditional fuzzy comprehensive analysis method requires a large number of experts to determine if a certain fault belongs to one of the five levels. On this basis, the hazard degree of each fault is calculated, and the result is obtained through normalization. $R_{1}=\left\{r_{1}, r_{2}, r_{3}, r_{4}, r_{5}\right\}$, where $R_{1}$ represents the judgment vector of the fault and $r_{i}$ indicates the membership degree of fault $i$ (the number of persons whose faults belong to the hazard category $i$ ). The calculation formula of membership degree is as follows:

$$
r_{i}=\frac{n_{i}}{n_{1}+n_{2}+n_{3}+n_{4}+n_{5}} .
$$

This method requires investigating numerous experts, is time-consuming and laborious, and does not consider the impact of the professional level of experts on the degree of harm; hence, the results obtained are inaccurate. Generally, the evaluation values given by most experts belong to a certain interval. This study calculates the membership degree based on the expert weight evaluation interval, that is, the evaluation interval is given to a few experts only to solve the shortcomings of having a large amount of research data. The calculation method of membership is as follows:

(a) If the interval value of the fault falls within a certain level range, then define $r_{i}=1$.

(b) If the interval falls within the range of $r_{i}$ and $r_{i+1}$, then the fault membership is defined to obey two levels. The calculation formula is as follows:

$$
\begin{aligned}
r_{i} & =\frac{2 \cdot i-Z_{i}^{L}}{Z_{i}^{U}-Z_{i}^{L}}, \\
r_{i+1} & =1-r_{i} .
\end{aligned}
$$

According to the membership table, it is transformed into the corresponding fuzzy judgment matrix $\{M 1, M 2, M 3$, M4, M5, M6, M7, M8, M9, M10\} because each factor has a different influence on the result. Therefore, a weight set must be established based on expert experience $\omega=\left[\omega_{1}, \omega_{2}, \omega_{3}\right]$. It is used to assess the low position of each factor in the rating. The weight must satisfy the normalization condition, namely,

$$
\omega_{1}+\omega_{2}+\omega_{3}=1
$$


The membership degree is transformed into a corresponding fuzzy judgment matrix, and the fuzzy comprehensive judgment set formula is obtained as

$$
G=\omega \mathrm{M} .
$$

The fuzzy evaluation set of each component fault is obtained based on the fuzzy evaluation set formula. The results need to be de-fuzzified to facilitate the comparison of relative hazards between faulty parts. The improved RPN value calculation formula is obtained as

$$
M_{\mathrm{RPN}}=G V^{T} .
$$

The fuzzy comprehensive evaluation method can integrate the experts' experience and knowledge into the evaluation matrix, which can make the results more scientific, reasonable, and accurate. The combination of group decision-making and fuzzy comprehensive evaluation method overcomes the inability to assign weights to severity, probability of occurrence, and detection difficulty, and in some cases, it is impossible to sort the shortcomings. The modified method is adopted to evaluate product failure modes. Given the evaluation results that are more in line with the actual use, it provides a reference for further improvement of reliability. Take the low temperature stop valve as an example for analysis and comparison.

\section{Case Study}

4.1. RPN Analysis of Cryogenic Stop Valve Based on Risk Priority Coefficient Method. The closing part (disc) of a cryogenic globe valve moves along the center line of the valve seat, and the working environment temperature is lower than $-150^{\circ} \mathrm{C}$. This type of valve is often used to cut off or adjust flow in many industries, such as LNG transport, and its reliability determines the safety of the entire system. A cryogenic globe valve is mainly composed of the valve body, valve clack assembly, valve stem, valve cover, and packaging. Its working principle is to rotate the hand wheel installed at the top of the valve stem of the stop valve to drive the valve stem to move up and down through the trapezoidal thread between the valve stem and the long neck pipe, thereby adjusting the opening and closing of the valve and the flow rate. The specific structure is shown in Figure 2.

The failure of the cryogenic globe valve is generally divided into failure of the sealing system and failure of the transmission system. Sealing system failures are primarily caused by multiple factors, such as seal wear or cracking and loosening of valve parts. Transmission system failures are relatively simple and mainly caused by transmission obstruction resulting from the deformation of the valve stem and its components. In accordance with the operating conditions of the low-temperature shut-off valve in the actual life survey of an enterprise, all the failure shadow modes of the low-temperature shut-off valve are obtained, and the RPN value is derived according to the calculation method of RPN, as shown in Table 3 and Figures 3 and 4 .

Table 3 and Figures 3 and 4 show that the RPN value of valve stem deformation and fracture (T9) is the largest, indicating that valve stem deformation and fracture exert the most harm in each failure mode. The valve clapper fastener is loose (T3), and the packing is worn loose. Moreover, the RPN values of T6 and the failure of the main seal (T7) are similar, indicating that $\mathrm{T} 3, \mathrm{~T} 6$, and $\mathrm{T} 7$ are the same in terms of hazard. This result is inconsistent with the results obtained in the actual use. It shows that the hazard analysis obtained by this algorithm is inaccurate.

4.2. RPN Analysis of Cryogenic Globe Valve Based on the Combination of Group Decision and Fuzzy Comprehensive Evaluation. Using the RPN analysis combining group decision-making and fuzzy comprehensive evaluation, three experts provide the interval scores of each failure mode severity $(\mathrm{S})$, occurrence degree $(\mathrm{O})$, and detection difficulty (D) for the 10 failure modes of the cryogenic shut-off valve. The details are shown in Table 4.

Given the presence of similarities and differences between individual experts and groups, according to the principle of group decision-making, the weights of experts are allocated to obtain the comprehensive evaluation interval of experts, which is then combined with fuzzy comprehensive evaluation to determine the membership degree of the three risk elements, as shown in Table 5.

On the basis of expert experience, the weight set of the three evaluation factors of severity, occurrence probability, and detection difficulty levels of the failure mode is given as $\omega=[0.3,0.5,0.2]$ [8]. The membership degree is transformed into a fuzzy evaluation matrix, and the fuzzy comprehensive evaluation set is de-fuzzified to obtain the low-temperature cut-off valve's RPN value by using the improved method. This value is then compared with the RPN value obtained by using RPN, as shown in Table 6 .

Table 6 shows that the RPN ranking after improvement and the RPN value ranking obtained by using RPN are different. This result is obtained because the RPN ranking before the improvement is mainly based on expert experience or judgment, and the ability levels of experts differ and are subjectively affected. The factor is large. By contrast, the RPN value obtained by the proposed method can be used to sort the 10 failure modes. The obtained fault sequence shows that the damage degree of valve disc wear and scratch (T1) is greater than the damage degree of valve stem deformation and fracture (T9). The working conditions are consistent, indicating that the hazard analysis of each failure mode obtained by the method of combining group decisionmaking and fuzzy comprehensive evaluation is highly accurate. 


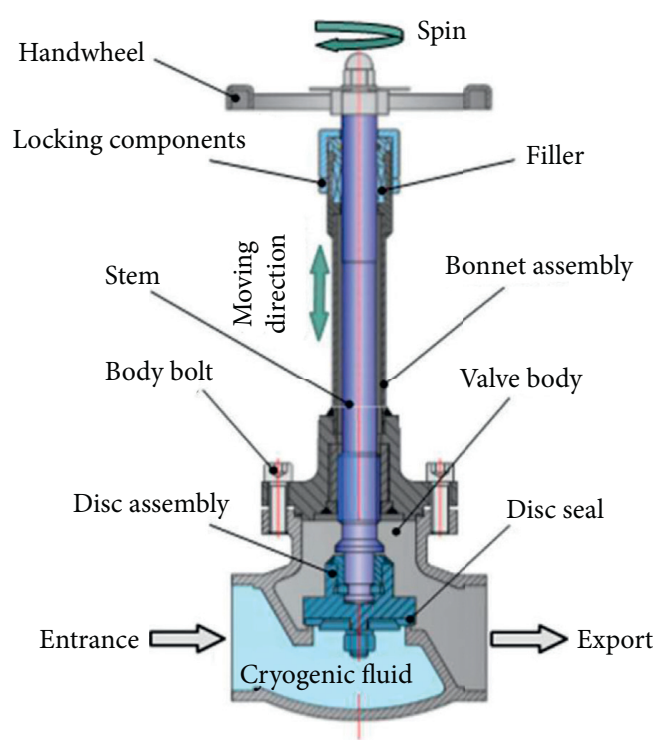

Figure 2: Diagram of the low-temperature globe valve structure.

TABLE 3: Cryogenic shut-off valve failure mode score table and RPN value.

\begin{tabular}{|c|c|c|c|c|c|}
\hline Subsystem & Failure mode & Severity $(S)$ & Occurrence $(\mathrm{O})$ & Difficulty of detection (D) & RPN \\
\hline \multirow{8}{*}{ Sealing system } & Disc wear and scratch (T1) & 5 & 8 & 5 & 200 \\
\hline & Stem wear (T2) & 6 & 3 & 6 & 108 \\
\hline & The disc fixing part is loose (T3) & 6 & 4 & 5 & 120 \\
\hline & Loose body bolts (T4) & 8 & 6 & 2 & 96 \\
\hline & Lock nut is loose (T5) & 4 & 6 & 2 & 48 \\
\hline & Packing wear and loose (T6) & 5 & 4 & 6 & 120 \\
\hline & Failure of the main gasket (T7) & 6 & 4 & 5 & 120 \\
\hline & Damaged and cracked valve body (T8) & 9 & 1 & 2 & 18 \\
\hline \multirow{2}{*}{ Transmission system } & Stem deformation and fracture (T9) & 7 & 5 & 6 & 210 \\
\hline & Valve stem assembly failure (T10) & 6 & 4 & 6 & 144 \\
\hline
\end{tabular}

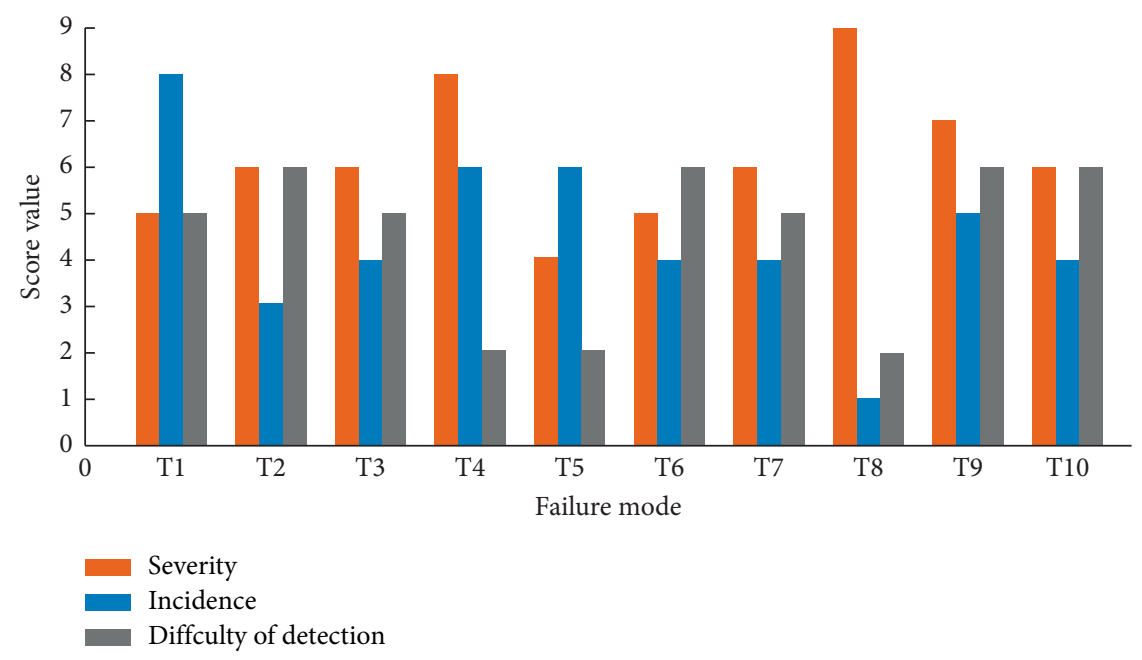

FIgURE 3: Low-temperature globe valve failure mode. 


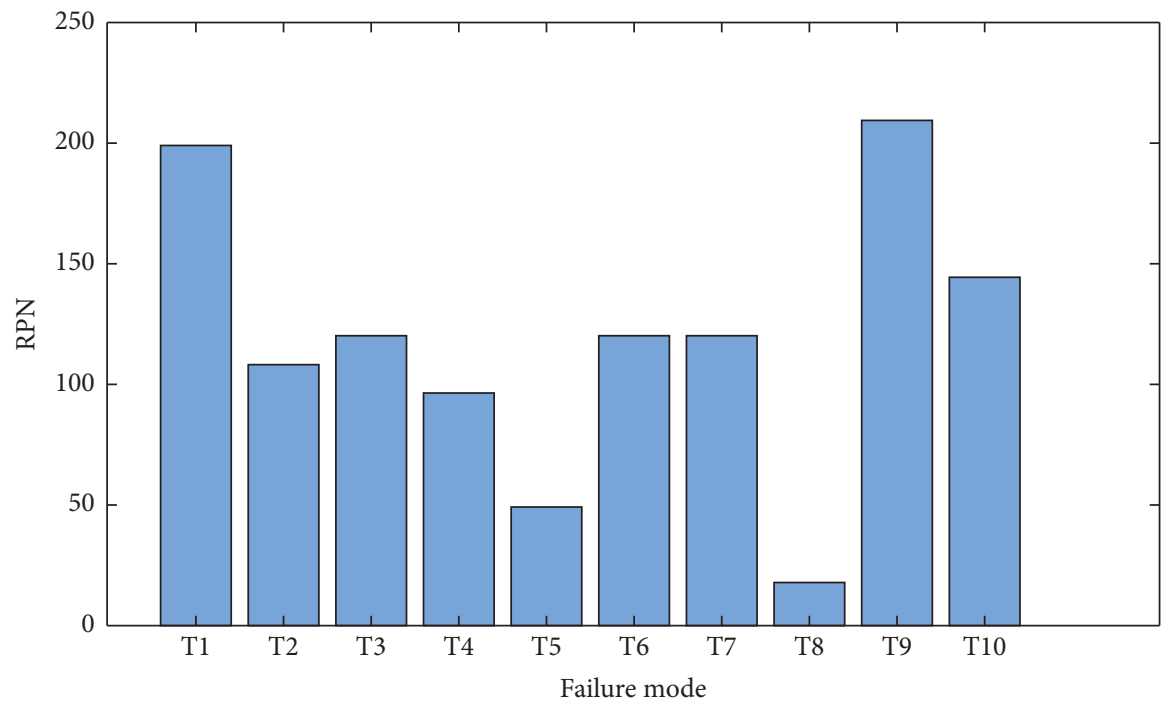

FiguRE 4: Low-temperature globe valve RPN table.

TABLE 4: Three experts' comprehensive evaluation values for severity (S), incidence (O), and detection difficulty (D).

\begin{tabular}{lccc}
\hline Failure mode & Expert 1 $(\mathrm{S} / \mathrm{O} / \mathrm{D})$ & Expert 2 $(\mathrm{S} / \mathrm{O} / \mathrm{D})$ & Expert 3 $(\mathrm{S} / \mathrm{O} / \mathrm{D})$ \\
\hline T1 & {$[4-4.5] /[8-8] /[4.5-5]$} & {$[4.2,6] /[8,8.5] /[5,5.3]$} & {$[4.3,4.3] /[7.3,8.2] /[5,5]$} \\
T2 & {$[6,6.5] /[3,3] /[5.2,6.5]$} & {$[6,6.3] /[2.6,4.4] /[5,6]$} & {$[5.5,6.4] /[3,3.5] /[5.5,6]$} \\
T3 & {$[6,7] /[4,5] /[4,5]$} & {$[6,6.5] /[3.7,4.6] /[4.5,5]$} & {$[5,6.8] /[4.1,5.5] /[4.6,5.5]$} \\
T4 & {$[7,8.3] /[6,6] /[1.5,2]$} & {$[7,8] /[5,6.8] /[1.8,3.6]$} & {$[7.5,8] /[6,6.5] /[2,2.5]$} \\
T5 & {$[3.5,4.9] /[6,6] /[2,2]$} & {$[3,4] /[5,6.8] /[1.85,3.2]$} & {$[3.8,4] /[5.5,6] /[1.9,2.8]$} \\
T6 & {$[4.5,5.4] /[4.5,4.5] /[5.5,6]$} & {$[5,5] /[4,5] /[5.5,6.4]$} & {$[4.2,6] /[5,5] /[5.8,6]$} \\
T7 & {$[6,6] /[4,5] /[5,5]$} & {$[5.8,6.2] /[4.5,5] /[4.6,5.2]$} & {$[5,6.8] /[4.5,4.5] /[4.5,5]$} \\
T8 & {$[9,9.5] /[0.5,1] /[1.8,2.5]$} & {$[8.2,9.1] /[1,1.9] /[1,2]$} & {$[8.7,9] /[1,2.3] /[1.5,2]$} \\
T9 & {$[6,7] /[4.5,5] /[6,6.5]$} & {$[7,7] /[4.6,5.5] /[6,6.3]$} & {$[5.8,7.6] /[5,6] /[5.5,6.4]$} \\
T10 & {$[6,7] /[4,4] /[5.2,6.6]$} & {$[6.5,7] /[3.5,4] /[5,6]$} & {$[5,6.8] /[3.4,5.2] /[5.5,6.4]$} \\
\hline
\end{tabular}

TABLE 5: Failure mode severity level (S), occurrence probability level (O), and detected difficulty level (D) membership.

\begin{tabular}{lccccccccccccccc}
\hline \multirow{3}{*}{ Failure serial number } & \multicolumn{4}{c}{ Severity level } & \multicolumn{4}{c}{ Probability level } & \multicolumn{4}{c}{ Detected difficulty level } \\
& 1 & 2 & 3 & 4 & 5 & 1 & 2 & 3 & 4 & 5 & 1 & 2 & 3 & 4 & 5 \\
\hline T1 & 0 & 0 & 1 & 0 & 0 & 0 & 0 & 0 & 0.52 & 0.48 & 0 & 0 & 1 & 0 & 0 \\
T2 & 0 & 0 & 0.32 & 0.68 & 0 & 0 & 1 & 0 & 0 & 0 & 0 & 0 & 0.81 & 0.19 & 0 \\
T3 & 0 & 0 & 0.27 & 0.73 & 0 & 0 & 0.06 & 0.94 & 0 & 0 & 0 & 0 & 1 & 0 & 0 \\
T4 & 0 & 0 & 0.88 & 0.12 & 0 & 0 & 0 & 0.41 & 0.59 & 0 & 0.25 & 0.75 & 0 & 0 & 0 \\
T5 & 0 & 0.66 & 0.34 & 0 & 0 & 0 & 0 & 0.68 & 0.32 & 0 & 0.11 & 0.89 & 0 & 0 & 0 \\
T6 & 0 & 0 & 1 & 0 & 0 & 0 & 0 & 1 & 0 & 0 & 0 & 0 & 0.79 & 0.21 & 0 \\
T7 & 0 & 0 & 0.54 & 0.46 & 0 & 0 & 0 & 1 & 0 & 0 & 0 & 0 & 1 & 0 & 0 \\
T8 & 0 & 0 & 0 & 0 & 1 & 1 & 0 & 0 & 0 & 0 & 0.82 & 0.18 & 0 & 0 & 0 \\
T9 & 0 & 0 & 0 & 1 & 0 & 0 & 0 & 1 & 0 & 0 & 0 & 0 & 0.26 & 0.74 & 0 \\
T10 & 0 & 0 & 0.11 & 0.89 & 0 & 0 & 0.5 & 0.5 & 0 & 0 & 0 & 0 & 0.70 & 0.30 & 0 \\
\hline
\end{tabular}

TABLE 6: Sorting table of the RPN values of each failure mode before and after using the improved CA.

\begin{tabular}{lcccc}
\hline Failure mode & RPN value & RPN sort & Improved RPN value & Improved RPN sort \\
\hline T1 & 200 & 2 & 3.74 & 1 \\
T2 & 108 & 5 & 2.742 & 8 \\
T3 & 120 & 4 & 3.189 & 3 \\
T4 & 96 & 6 & 3.081 & 5 \\
T5 & 48 & 7 & 2.74 & 9 \\
T6 & 120 & 4 & 3.042 & 7 \\
T7 & 120 & 4 & 3.138 & 4 \\
T8 & 18 & 8 & 2.236 & 10 \\
T9 & 210 & 1 & 3.448 & 2 \\
T10 & 144 & 3 & 3.077 & 6 \\
\hline
\end{tabular}




\section{Conclusions}

This study used a hazard assessment method that combines group decision-making and fuzzy comprehensive evaluation. A reliability analysis was conducted on the hazard of each failure mode of a cryogenic shut-off valve. The following conclusions were obtained:

(1) Using the degree of similarity and difference between experts in group decision-making to assign expert weights can truly and objectively reflect the hazards of each failure mode and overcome the influence of experts' subjective factors on the evaluation results. The method of fuzzy comprehensive evaluation weighs the three risk elements and ranks the comprehensive evaluation interval simultaneously. Hence, the failure mode ranking is highly accurate.

(2) A criticality analysis of the cryogenic shut-off valve was conducted. The weak links of reliability were identified, and the rationality and feasibility of the method were verified.

(3) The CA improvement method is mainly used to analyze the hazard posed by low-temperature shut-off valves, which can intuitively reveal the degree of harm of the product failure mode, and the implementation of improvement measures is taken based on its results. It provides technical support for evaluating the safety and reliability of special applications such as air separation and LNG transportation.

(4) This paper uses a combined group decision-making method to reduce the influence of subjective factors of experts. In the future, it can also be combined with the Pythagorean fuzzy entropy measure [12], based on the weighted average and the ordered weighted average operator, to explore the establishment of new attribute weight methods.

(5) The relationship between membership degree and nonmembership degree used in this paper meets the conditions of Pythagorean fuzzy set that the sum of the membership degree and nonmembership degree is less than or equal to 1 . In the future [13], the methods of the Pythagorean theorem fuzzy interaction operator and the weighted Pythagorean theorem fuzzy interaction operator should be combined to conduct a more indepth study on the relationship between membership and nonmembership.

\section{Data Availability}

The data used to support the findings of the study are available from the corresponding author upon request.

\section{Conflicts of Interest}

The authors declare that they have no conflicts of interest.

\section{Acknowledgments}

This research was supported by the National Natural Science Foundation of China (Grant no. 51475367), National Science and Technology Major Project of China (Grant no. 2013ZX04009011), Natural Science Foundation of Department of Education of Shaanxi Province (Grant no. 2018JM5113), and Key Laboratory Project of Shaanxi Education Department (Grant no. 17JS093).

\section{References}

[1] L. Qiang, L. Xie, H. Li, F. Zhang, and J. Song, "Precision gear manufacturing process optimization priority analysis based on fuzzy comprehensive hierarchical evaluation method," Acta Armamentarii, vol. 38, no. 4, pp. 750-757, 2017.

[2] C. Dai, X. Wang, X. Zhang, and L. Wang, "Electro-hydraulic servo valve FMECA based on fuzzy comprehensive evaluation," Journal of Beijing University of Aeronautics and Astronautics, vol. 37, no. 12, pp. 1575-1578, 2011.

[3] K.-H. Chang, "Evaluate the orderings of risk for failure problems using a more general RPN methodology," Microelectronics Reliability, vol. 49, no. 12, pp. 1586-1596, 2009.

[4] K. S. Chen, C. C. Wang, C. H. Wang, and C. F. Huang, "Application of RPN analysis to parameter optimization of passive components," Microelectronics Reliability, vol. 50, no. 12, pp. 2012-2019, 2010.

[5] U. K. Bhattacharjee, V. Dey, and U. K. Mandal, "Risk assessment by failure mode and effects analysis (FMEA) using an interval number based logistic regression model," Safety Science, vol. 132, Article ID 104967, 2020.

[6] V. R. Renjith, M. Jose Kalathil, P. H. Kumar et al., "Fuzzy FMECA (failure mode effect and criticality analysis) of LNG storage facility," Journal of Loss Prevention in the Process Industries, vol. 56, pp. 537-547, 2018.

[7] M. Catelani, L. Ciani, D. Galar et al., "Risk assessment of a wind turbine: a new FMECA-based tool with RPN threshold estimation," IEEE Access, vol. 8, pp. 20181-20190, 2020.

[8] X. Wang, G. Shen, Y. Zhang et al., "RPN analysis of key components of machining center based on group decision and multiple assignment methods," Journal of Jilin University (Engineering and Technology Edition), vol. 6, pp. 1630-1635, 2011.

[9] A. Akram and A. Adeel, "Novel TOPSIS method for group decision-making based on hesitant m-polar fuzzy model," Journal of Intelligent \& Fuzzy Systems, vol. 37, no. 6, pp. 8077-8096, 2019.

[10] H. Akram, F. Ilyas, and H. Garg, "Multi-criteria group decision making based on ELECTRE I method in Pythagorean fuzzy information," Soft Computing, vol. 24, no. 5, pp. 3425-3453, 2020.

[11] S. Wan, "Method for determining the weight of interval-type multi-attribute group experts," Journal of Applied Mathematics and Computational Mathematics, vol. 22, no. 2, pp. 109-116, 2008.

[12] L. Wang, H. Garg, and N. Li, "Pythagorean fuzzy interactive Hamacher power aggregation operators for assessment of express service quality with entropy weight," Soft Computing, vol. 25, no. 2, pp. 973-993, 2020.

[13] L. Wang and N. Li, "Pythagorean fuzzy interaction power Bonferroni mean aggregation operators in multiple attribute decision making," International Journal of Intelligent Systems, vol. 35 , no. $1,2020$. 